\title{
Generalized parton distributions in an AdS/QCD hard-wall model
}

\author{
Alfredo Vega ${ }^{1}$, Ivan Schmidt ${ }^{1}$, Thomas Gutsche ${ }^{2}$ and Valery E. Lyubovitskij ${ }^{2 *}$ \\ ${ }^{1}$ Departamento de Física y Centro Científico y Tecnológico de Valparaíso, \\ Universidad Técnica Federico Santa María, \\ Casilla 110-V, Valparaíso, Chile \\ 2 Institut für Theoretische Physik, Universität Tübingen, \\ Kepler Center for Astro and Particle Physics, \\ Auf der Morgenstelle 14, D-72076 Tübingen, Germany
}

(Dated: May 26, 2021)

\begin{abstract}
We use a matching procedure of sum rules relating the electromagnetic form factors to generalized parton distributions (GPDs) and AdS modes. In this way, in the framework of an AdS/QCD hardwall model, the helicity-independent GPDs of quarks for the nucleon in the zero skewness case are calculated.

PACS numbers: 11.10.Kk,12.38.Lg,13.40.Gp,14.20.Dh

Keywords: Nucleons, generalized parton distributions, AdS/CFT correspondence, holographical model
\end{abstract}

\section{INTRODUCTION}

Light Front Holography (LFH) [1-3] is a semiclassical approximation to QCD based on the gauge/gravity duality that provides a precise mapping of the string modes $\Phi(z)$ in the anti-de Sitter (AdS) fifth dimension $z$ to the hadron light-front wave functions (LFWFs) in physical space-time. This approach has been successfully applied to the description of the mass spectrum of mesons and baryons (e.g. reproducing the Regge trajectories), the pion leptonic constant, the electromagnetic form factors of pion and nucleons, etc. [4]-12]. The mapping that allows to relate AdS modes to a LFWF is obtained by matching certain matrix elements (e.g. the electromagnetic pion form factor) in the two approaches - string theory in AdS and Light-Front QCD in Minkowski spacetime.

The same idea can be used to calculate the generalized parton distributions (GPDs) of the nucleon [9 11], which encode important information about the hadronic structure. This is a new subject in the application of the gauge/gravity correspondence to hadronic properties in the strong coupling limit where QCD cannot be used in a straightforward way.

GPDs are of nonperturbative nature and therefore cannot be easily calculated from Quantum Chromodynamics (QCD). We have essentially three ways to access the GPDs (for reviews see e.g. [13, 14]): extraction from the experimental measurement of hard processes, a direct calculation in the context of lattice QCD and different phenomenological models and methods. An example of the latter procedure is based on a parametrization of the

\footnotetext{
* On leave of absence from Department of Physics, Tomsk State University, 634050 Tomsk, Russia
}

quark wave functions/GPDs using constraints imposed by sum rules [15, 16, which relate the parton distributions to nucleon electromagnetic form factors (some examples of this procedure can be found e.g. in [17 19]).

In a previous work 9 , we showed how to obtain the quark GPDs of the nucleon in a soft-wall model. We applied a matching procedure similar to the one used in LFH applications. In this paper we study helicityindependent GPDs in the hard-wall holographical model suggested and developed in Refs. [20, 21, and then applied for the nucleon sector in [22].

In general, soft-wall models have some advantages compared to hard-wall models. In particular, the hadronic mass spectrum in soft-wall models has Reggelike behavior, and most of the calculations can be done analytically. But, as can be seen e.g. in [22, in hard-wall models the obtained nucleon form factors are in better agreement with data. This makes it worthwhile to consider the GPDS in the context of holographic hard-wall models. Additionally, we would like to discuss the high momentum transfer limit $\left(Q^{2} \rightarrow \infty\right)$ for the physical quantities of our calculations. Note that in this limit the soft and hard-wall approaches have the same behavior: the electromagnetic probe propagating in the AdS space decouples from the dilaton. Something similar happens in the case of the pion form factor [4].

In this work we consider a procedure similar to the one discussed in 9 for the soft-wall case, i.e. we perform a matching of the nucleon electromagnetic form factors considering two main ideas: we use sum rules, derived in QCD [15, 16, which contain the GPDs for the valence quarks, and we consider specific integral representations obtained in the AdS/QCD hard-wall model 22. As result of the matching we obtain expressions for the nonforward parton densities [25] $H_{v}^{q}(x, t)=H^{q}(x, 0, t)+$ $H^{q}(-x, 0, t)$ and $E_{v}^{q}(x, t)=E^{q}(x, 0, t)+E^{q}(-x, 0, t)-$ flavor combinations of the GPDs (or valence GPDs), us- 
ing information obtained on the AdS side.

The paper is organized as follows. First, in Sec. II we review the sum rules that relate the GPDs to the nucleon form factors. After that, in Sec. III we summarize the main results of Ref. 22 obtained for nucleon form factors in the framework of the hard-wall model. In Sec. IV we outline the matching procedure used in 9] now applied to the hard-wall case. In Sec. $\mathrm{V}$ we consider the high $Q^{2}$ limit, noticing that we get the same behavior as in the soft-wall case. Finally, we draw our conclusions in Sec. VI.

\section{GPDS AND ELECTROMAGNETIC FORM FACTORS FOR THE NUCLEON}

The nucleon electromagnetic form factors $F_{1}^{N}$ and $F_{2}^{N}$ ( $N=p, n$ correspond to proton and neutron) are conventionally defined by the matrix element of the electromagnetic current as

$$
\left\langle p^{\prime}\left|J^{\mu}(0)\right| p\right\rangle=\bar{u}\left(p^{\prime}\right)\left[\gamma^{\mu} F_{1}^{N}(t)+\frac{i \sigma^{\mu \nu}}{2 m_{N}} q_{\nu} F_{2}^{N}(t)\right] u(p),
$$

where $q=p^{\prime}-p$ is the momentum transfer; $m_{N}$ is the nucleon mass; $F_{1}^{N}$ and $F_{2}^{N}$ are the Dirac and Pauli form factors, which are normalized to electric charge $e_{N}$ and anomalous magnetic moment $k_{N}$ of the corresponding nucleon: $F_{1}^{N}(0)=e_{N}$ and $F_{2}^{N}(0)=k_{N}$.

The sum rules relating the electromagnetic form factors and the GPDs read as [15, 16, 25]

$$
\begin{aligned}
& F_{1}^{p}(t)=\int_{0}^{1} d x\left(\frac{2}{3} H_{v}^{u}(x, t)-\frac{1}{3} H_{v}^{d}(x, t)\right), \\
& F_{1}^{n}(t)=\int_{0}^{1} d x\left(\frac{2}{3} H_{v}^{d}(x, t)-\frac{1}{3} H_{v}^{u}(x, t)\right), \\
& F_{2}^{p}(t)=\int_{0}^{1} d x\left(\frac{2}{3} E_{v}^{u}(x, t)-\frac{1}{3} E_{v}^{d}(x, t)\right), \\
& F_{2}^{n}(t)=\int_{0}^{1} d x\left(\frac{2}{3} E_{v}^{d}(x, t)-\frac{1}{3} E_{v}^{u}(x, t)\right) .
\end{aligned}
$$

Here we restrict to the contribution of the $u, d$ quarks and respective antiquarks, while the presence of the heavier strange and charm quark constituents is not considered.

\section{ELECTROMAGNETIC FORM FACTORS FOR THE NUCLEON IN THE ADS/QCD HARD-WALL MODEL}

Here we outline the relevant results for the nucleon form factors using a hard wall AdS/QCD model as obtained by Abidin and Carlson [22]. In this model a cut off $z_{0}$ is introduced in the AdS space, which leads to a breaking of the conformal invariance and thereby simulates confinement. The AdS metric is specified as

$$
d s^{2}=g_{M N} d x^{M} d x^{N}=\frac{1}{z^{2}}\left(\eta_{\mu \nu} d x^{\mu} d x^{\nu}-d z^{2}\right)
$$

where $\mu, \nu=0,1,2,3 ; \eta_{\mu \nu}=\operatorname{diag}(1,-1,-1,-1)$ is the Minkowski metric tensor and $z$ is the holographical coordinate running from zero to $z_{0}$.

The relevant terms in the AdS/QCD action which generate the nucleon form factors are [22]:

$$
\begin{aligned}
S & =\int d^{4} x d z \sqrt{g}\left(\bar{\Psi} e_{A}^{M} \Gamma^{A} V_{M} \Psi\right. \\
& \left.+\frac{i}{2} \eta_{S, V} \bar{\Psi} e_{A}^{M} e_{B}^{N}\left[\Gamma^{A}, \Gamma^{B}\right] F_{M N}^{(S, V)} \Psi\right),
\end{aligned}
$$

where the basic blocks of the AdS/QCD model are defined as: $g=\left|\operatorname{det} g_{M N}\right| ; \Psi$ and $V_{M}$ are the 5D Dirac and vector fields dual to the nucleon and electromagnetic fields, respectively; $F_{M N}=\partial_{M} V_{N}-\partial_{N} V_{M} ; \Gamma^{A}=$ $\left(\gamma^{\mu},-i \gamma^{5}\right) ; e_{A}^{M}=z \delta_{A}^{M}$ is the inverse vielbein; $\eta_{S, V}$ are the couplings constrained by the anomalous magnetic moment of the nucleon (see below). Here the indices $S, V$ denote isoscalar and isovector contributions to the electromagnetic form factors.

Finally, results for the nucleon form factors in AdS/QCD are given in 22. For the proton we get:

$$
\begin{aligned}
& F_{1}^{p}\left(Q^{2}\right)=C_{1}\left(Q^{2}\right)+\eta_{p} C_{2}\left(Q^{2}\right) \\
& F_{2}^{p}\left(Q^{2}\right)=\eta_{p} C_{3}\left(Q^{2}\right)
\end{aligned}
$$

and for the neutron

$$
\begin{aligned}
& F_{1}^{n}\left(Q^{2}\right)=\eta_{n} C_{2}\left(Q^{2}\right) \\
& F_{2}^{n}\left(Q^{2}\right)=\eta_{n} C_{3}\left(Q^{2}\right),
\end{aligned}
$$

In above expressions we have $\eta_{p}=\left(\eta_{S}+\eta_{V}\right) / 2, \eta_{n}=$ $\left(\eta_{S}-\eta_{V}\right) / 2, Q^{2}=-t$ and the $C_{i}$ are integrals defined by:

$$
\begin{aligned}
C_{1}\left(Q^{2}\right) & =\int_{0}^{z_{0}} d z \frac{V(Q, z)}{2 z^{3}}\left[\psi_{L}^{2}(z)+\psi_{R}^{2}(z)\right], \\
C_{2}\left(Q^{2}\right) & =\int_{0}^{z_{0}} d z \frac{\partial_{z} V(Q, z)}{2 z^{2}}\left[\psi_{L}^{2}(z)-\psi_{R}^{2}(z)\right], \\
C_{3}\left(Q^{2}\right) & =\int_{0}^{z_{0}} d z \frac{2 m_{N} V(Q, z)}{z^{2}} \psi_{L}(z) \psi_{R}(z),
\end{aligned}
$$

where the coordinate $z$ runs from zero to a maximum value $z_{0}=(0.245 \mathrm{GeV})^{-1} \simeq 0.81 \mathrm{fm}$. The latter value was fixed in Ref. 22] by the $\rho$-meson and nucleon masses. The nucleon magnetic moments are expressed in terms of $\eta_{p}$ and $\eta_{n}$ as $\mu_{p}=1+\eta_{p} C_{3}(0)$ and $\mu_{p}=\eta_{n} C_{3}(0)$. 

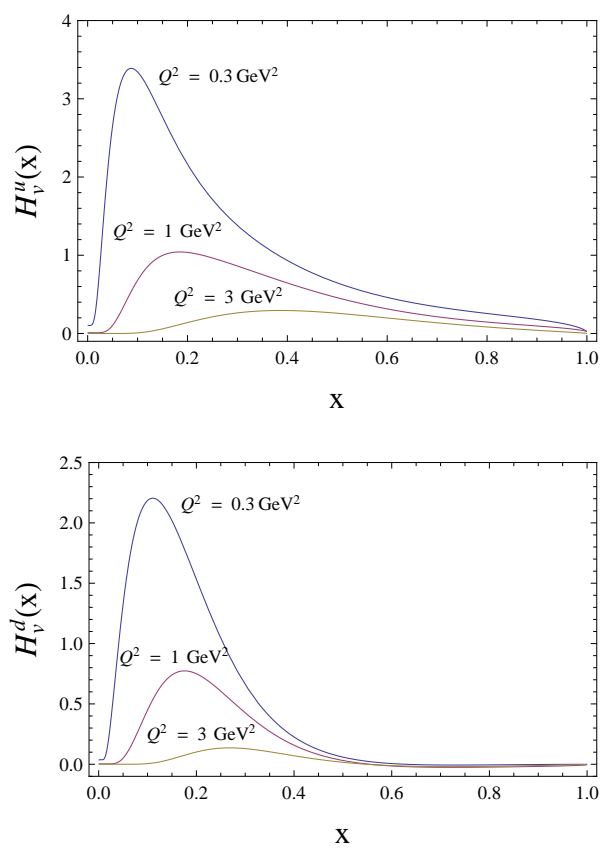

FIG. 1: $H_{v}^{q}(x)$ in the holographical model for different values of $Q^{2}$.
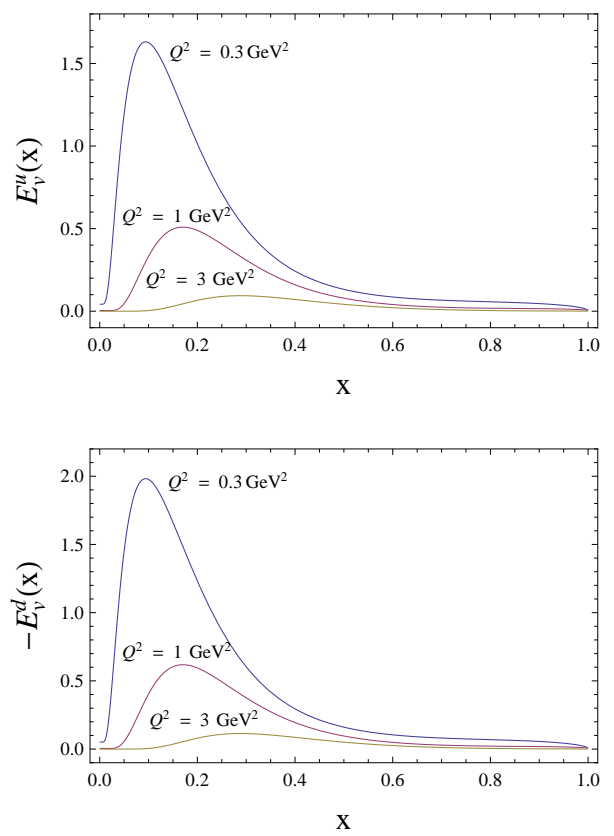

FIG. 2: $E_{v}^{q}(x)$ in the holographical model for different values of $Q^{2}$.

According to 22], $\psi_{L}(z)$ and $\psi_{R}(z)$ are

$$
\begin{aligned}
& \psi_{L}(z)=\frac{\sqrt{2} z^{2} J_{2}\left(m_{N} z\right)}{z_{0} J_{2}\left(m_{N} z_{0}\right)} \\
& \psi_{R}(z)=\frac{\sqrt{2} z^{2} J_{1}\left(m_{N} z\right)}{z_{0} J_{2}\left(m_{N} z_{0}\right)}
\end{aligned}
$$

and for the bulk-to-boundary propagator of the vector field in the axial gauge we have [21, 22]

$$
V(Q, z)=Q z\left[\frac{K_{0}\left(Q z_{0}\right)}{I_{0}\left(Q z_{0}\right)} I_{1}(Q z)+K_{1}(Q z)\right],
$$

where $J_{\nu}, I_{\nu}, K_{\nu}$ are the Bessel and modified Bessel functions [26. Note that expressions for the nucleon form factors in AdS/QCD can be obtained after integration over the variable $z$. Here we are interested in obtaining GPDs using a matching procedure that we will discuss in the next section. In this case we follow the formalism developed in Ref. [9].

Fixing the nucleon magnetic moments $\mu_{p}=2.793$ and $\mu_{n}=-1.913$ by appropriate choice of the nonminimal couplings $\eta_{p}=0.448$ and $\eta_{n}=-0.478$ 22. one can get a reasonable description of the nucleon form factors and their slopes. In particular, the electromagnetic radii of the nucleon are given in the hard-wall model by:

$$
\begin{aligned}
\left\langle r_{E}^{2}\right\rangle^{p} & =-6\left(C_{1}^{\prime}(0)+\eta_{p} C_{2}^{\prime}(0)-\eta_{p} \frac{C_{3}(0)}{4 m_{N}^{2}}\right), \\
\left\langle r_{E}^{2}\right\rangle^{n} & =-6 \eta_{n}\left(C_{2}^{\prime}(0)-\frac{C_{3}(0)}{4 m_{N}^{2}}\right), \\
\left\langle r_{M}^{2}\right\rangle^{p} & =-6 \frac{C_{1}^{\prime}(0)+\eta_{p} C_{2}^{\prime}(0)+\eta_{p} C_{3}^{\prime}(0)}{1+\eta_{p} C_{3}(0)}, \\
\left\langle r_{M}^{2}\right\rangle^{n} & =-6 \frac{C_{2}^{\prime}(0)+C_{3}^{\prime}(0)}{C_{3}(0)}
\end{aligned}
$$

where

$$
\begin{aligned}
& C_{1}^{\prime}(0)=-\frac{1}{8} \int_{0}^{z_{0}} \frac{d z}{z}\left(1-2 \log \left(\frac{z}{z_{0}}\right)\right)\left[\psi_{L}^{2}(z)+\psi_{R}^{2}(z)\right] \\
& C_{2}^{\prime}(0)=\frac{1}{2} \int_{0}^{z_{0}} \frac{d z}{z^{2}} \log \left(\frac{z}{z_{0}}\right)\left[\psi_{L}^{2}(z)-\psi_{R}^{2}(z)\right] \\
& C_{3}^{\prime}(0)=-\frac{m_{N}}{2} \int_{0}^{z_{0}} d z\left(1-2 \log \left(\frac{z}{z_{0}}\right)\right) \psi_{L}(z) \psi_{R}(z)
\end{aligned}
$$

Note that in the context of AdS/QCD the nucleon charge radii have been discussed before in [22]. Here we add the results for the magnetic radii. Numerical results for the slopes of the nucleon form factors compare rather well with data:

$$
\begin{aligned}
\left\langle r_{E}^{2}\right\rangle^{p} & =0.829 \mathrm{fm}^{2} \text { (our), } \quad 0.766 \mathrm{fm}^{2} \text { (data) } \\
\left\langle r_{E}^{2}\right\rangle^{n} & =-0.101 \mathrm{fm}^{2} \text { (our), } \quad-0.116 \mathrm{fm}^{2} \text { (data) } \\
\left\langle r_{M}^{2}\right\rangle^{p} & =0.756 \mathrm{fm}^{2} \text { (our), } \quad 0.731 \mathrm{fm}^{2} \text { (data), } \\
\left\langle r_{M}^{2}\right\rangle^{n} & =0.768 \mathrm{fm}^{2} \text { (our), } \quad 0.762 \mathrm{fm}^{2} \text { (data) }
\end{aligned}
$$

\section{GPDS AND ADS MODES IN A SOFT-WALL MODEL}

As is explained in 9, the matching procedure is based on the use of an integral representation for the bulk- 
to-boundary propagator. In the present case, according to 15 , we need an integral representation both for $I_{1}(Q z)$ [27] and $K_{1}(Q z)$ (see Appendix),

$$
I_{1}(Q z)=\frac{2 z Q}{\pi} \int_{0}^{1} d x \sqrt{1-x^{2}} \cosh (x z Q)
$$

$K_{1}(Q z)=\frac{z}{Q} \int_{0}^{1} \frac{d x}{(1-x)^{2}} \exp \left(-\frac{Q^{2}(1-x)}{4 x}-\frac{z^{2} x}{1-x}\right)$.

With these representations it is possible to calculate some of the GPDs starting from $V(Q, z)$ and $\psi_{R / L}(z)$. The procedure consists in replacing $\sqrt{15}$, using $\sqrt{19}$ and $(20)$ in 12 , and after performing the $z$ integration, each $C_{i}$ expression is now an integral in $x$, which runs from 0 to 1 . Using this technique in Eqs. (8)-(11), and comparing with Eqs. (2)-(5) we can identify the GPDs involved in the sum rules.

The procedure summarized in the last paragraph was applied in the soft-wall model to obtain analytical expressions 9] for some GPDs. Unfortunately here it is not possible to get analytical results, although numerical calculations can be done without problems using Mathematica. Numerical results for some $Q^{2}$ values are shown in Fig.1 and Fig.2.

\section{NUCLEON GPDS IN IMPACT SPACE}

Other interesting quantities to consider are the nucleon GPDs in impact space. As shown by Burkardt [28, 29], the GPDs in momentum space are related to impact parameter dependent parton distributions via a Fourier transform. GPDs in impact space give access to the distribution of partons in the transverse plane, which is important for the understanding of the nucleon structure.

As an example we consider the nucleon GPDs in impact space [17, 28, 30, as

$$
\begin{aligned}
q\left(x, \mathbf{b}_{\perp}\right) & =\int \frac{d^{2} \mathbf{k}_{\perp}}{(2 \pi)^{2}} H_{q}\left(x, \mathbf{k}_{\perp}^{2}\right) e^{-i \mathbf{b}_{\perp} \mathbf{k}_{\perp}} \\
e^{q}\left(x, \mathbf{b}_{\perp}\right) & =\int \frac{d^{2} \mathbf{k}_{\perp}}{(2 \pi)^{2}} E_{q}\left(x, \mathbf{k}_{\perp}^{2}\right) e^{-i \mathbf{b}_{\perp} \mathbf{k}_{\perp}}
\end{aligned}
$$

and the parton charge $\left(\rho_{E}\left(\mathbf{b}_{\perp}\right)\right)$ and magnetization $\left(\rho_{M}\left(\mathbf{b}_{\perp}\right)\right)$ densities in transverse impact space

$$
\begin{aligned}
\rho_{E}\left(\mathbf{b}_{\perp}\right) & =\sum_{q} e_{q} \int_{0}^{1} d x q\left(x, \mathbf{b}_{\perp}\right), \\
\rho_{M}\left(\mathbf{b}_{\perp}\right) & =\sum_{q} e_{q} \int_{0}^{1} d x e^{q}\left(x, \mathbf{b}_{\perp}\right) .
\end{aligned}
$$

Results for both flavors of $q\left(x, \mathbf{b}_{\perp}\right)$ with $\mathrm{x}=0.1$ are shown in Fig. 3. In Fig. 4 we give the results for the parton charge and magnetization densities in transverse impact space.

\section{COMPARISON WITH THE SOFT-WALL MODEL IN THE HIGH $Q^{2}$ LIMIT}

In the high $Q^{2}$ limit only the second term in 15 is important with $V(Q, z) \rightarrow Q z K_{1}(Q z)$. Since the Bessel function $K_{1}(Q z)$ decreases exponentially in the high $Q^{2}$ limit, we only need to consider its value near $z=0$. We therefore need $\psi_{L}(z)$ and $\psi_{R}(z)$ near $z=0$, i.e.

$$
\begin{aligned}
& \psi_{L}(z)=\frac{f_{N} m_{N}}{4} z^{4} \\
& \psi_{R}(z)=f_{N} z^{3}
\end{aligned}
$$

where

$$
f_{N}=\frac{m_{N}}{z_{0} J_{2}\left(m_{N} z_{0}\right) \sqrt{2}}
$$

Note that in the high $Q^{2}$ limit the integral that appears in (12) has the same form as in the soft-wall case. To clarify this point we use some results from [9, 22].

In the soft-wall case the $C_{i}\left(Q^{2}\right)$ are defined as (adding a super-script SW to avoid possible confusion): 

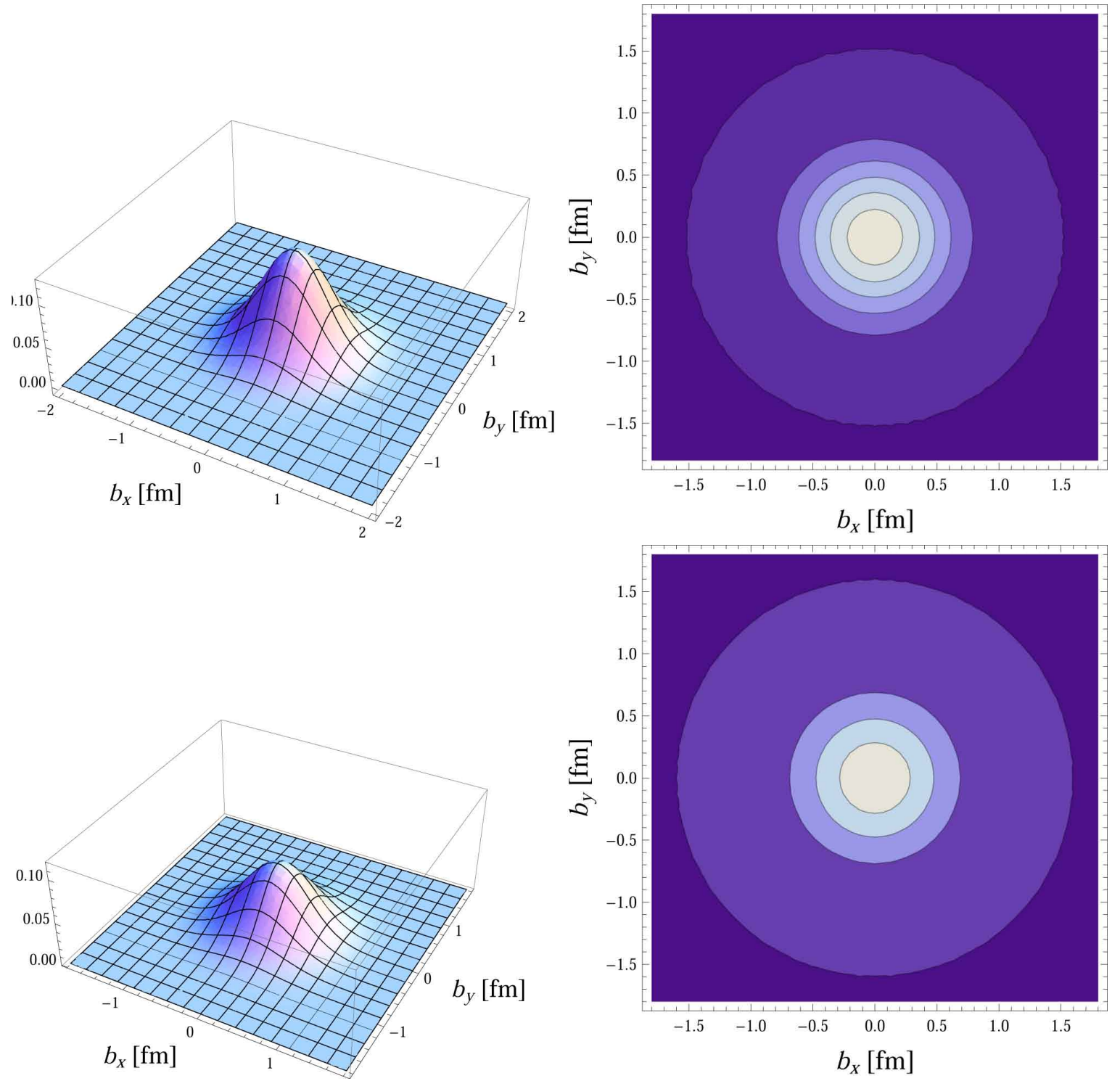

FIG. 3: Plots for $q\left(x, \mathbf{b}_{\perp}\right)$. The upper ones correspond to $u\left(x, \mathbf{b}_{\perp}\right)$ and the lower to $d\left(x, \mathbf{b}_{\perp}\right)$. Both cases are for $x=0.1$.

$$
\begin{aligned}
& C_{1}^{S W}\left(Q^{2}\right)=\int_{0}^{\infty} d z e^{-\varphi(z)} \frac{V(Q, z)}{2 z^{3}}\left[\left(\psi_{L}^{S W}(z)\right)^{2}+\left(\psi_{R}^{S W}(z)\right)^{2}\right], \\
& C_{2}^{S W}\left(Q^{2}\right)=\int_{0}^{\infty} d z e^{-\varphi(z)} \frac{\partial_{z} V(Q, z)}{2 z^{2}}\left[\left(\psi_{L}^{S W}(z)\right)^{2}-\left(\psi_{R}^{S W}(z)\right)^{2}\right], \\
& C_{3}^{S W}\left(Q^{2}\right)=\int_{0}^{\infty} d z e^{-\varphi(z)} \frac{2 m_{N} V(Q, z)}{z^{2}} \psi_{L}^{S W}(z) \psi_{R}^{S W}(z),
\end{aligned}
$$

where $\varphi(z)$ is the dilaton field. $\psi_{L}^{S W}(z)$ and $\psi_{R}^{S W}(z)$ are the Kaluza-Klein modes (normalizable wave functions), which are dual to the left- and right-handed nucleon fields:

$$
\psi_{L}^{S W}(z)=\kappa^{3} z^{4}, \quad \psi_{R}^{S W}(z)=\kappa^{2} z^{3} \sqrt{2}
$$



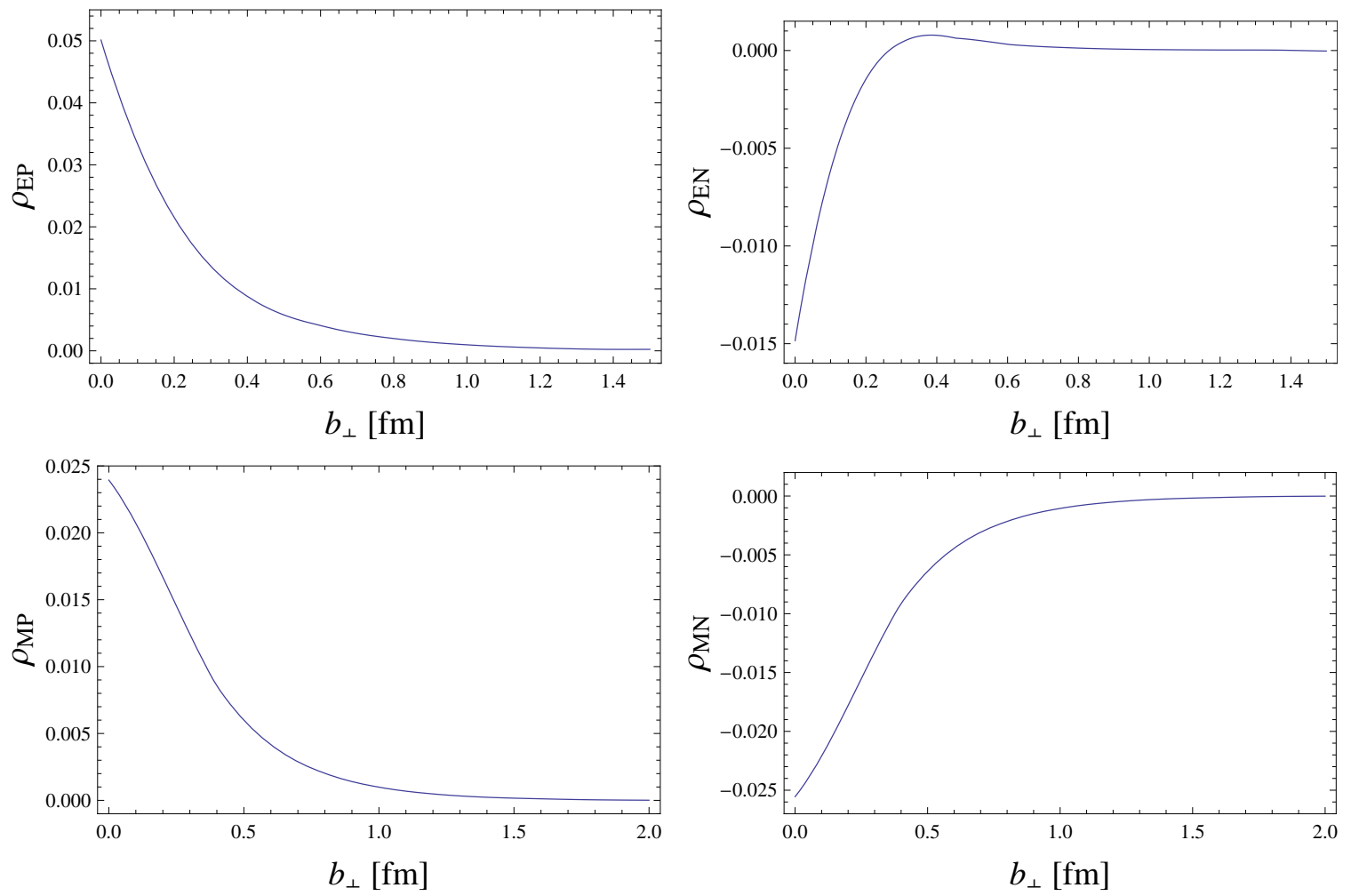

FIG. 4: Parton charge $\left(\rho_{E}\left(\mathbf{b}_{\perp}\right)\right)$ and magnetization $\left(\rho_{M}\left(\mathbf{b}_{\perp}\right)\right)$ densities in transverse impact space.

and which have the same form as in 23 and 244 . We also need to take the high $Q^{2}$ limit of the axial gauge vector field bulk-to-boundary propagator in the soft-wall model:

$$
V^{S W}(Q, z)=\Gamma\left(1+\frac{Q^{2}}{4 \kappa^{2}}\right) U\left(\frac{Q^{2}}{4 \kappa^{2}}, 0, \kappa^{2} z^{2}\right)
$$

where $\Gamma(a)$ and $U(a, b, z)$ are the Gamma and Kummer functions 26. As can be seen in 4, we have $V^{S W}(Q, z) \rightarrow z Q K_{1}(z Q)$ for high $Q^{2}$, i.e in this limit $\left(Q^{2} \gg 4 \kappa^{2}\right)$ the current decouples from the dilaton field. Additionally, since we only need the expressions near $z=0$ we can take $e^{-\varphi(z)}=1$, where we are considering a quadratic dilaton $\varphi(z)=\kappa^{2} z^{2}$. With all these ingredients we can see that

$$
C_{i}^{H W}=C_{i}^{S W}, \quad \operatorname{High} Q^{2} \text { limit. }
$$

Note that the parameters of the soft-wall model are the same as those used in 9 , 22, i.e. $\kappa=350 \mathrm{MeV}$, $\eta_{p}=0.224, \eta_{n}=-0.239$, which were fixed in order to reproduce the mass $m_{N}=2 \kappa \sqrt{2}$ and the anomalous magnetic moments of the nucleons.

\section{CONCLUSIONS}

We determined numerically the generalized parton distributions of nucleons in an AdS/QCD formalism based on a hard-wall scenario of conformal symmetry breaking (holographic hard wall model). We considered a procedure similar to the one used in some applications of LFH and applied previously in 9 for the soft-wall case. Although soft-wall models in general give a better hadronic Regge spectrum than in the hard-wall case, the latter sometimes is more successful in the description of hadronic form factors. It provides a strong motivation for the present work. Of course, the soft-wall model can also be improved by considering more complicated dilaton profiles.

Another interesting fact is that in the high $Q^{2}$ limit the form factors have the same form in both approaches (hard- and soft-wall models), i.e the dilaton decouples in this limit, a property noted in 4 for the pion form factor. So the GPDs must be equal in both kinds of holographical approaches at high $Q^{2}$.

\section{Acknowledgments}

This work was supported by FONDECYT (Chile) under Grants No. 3100028 and No. 1100287. This research is also part of the Federal Targeted Program "Scientific and scientific-pedagogical personnel of innovative Russia" Contract No. 02.740.11.0238. 
Appendix A: The integral representation used for $K_{1}(z Q)$

We start by considering the integral (C7) from 4 ]

$$
K_{\nu}(z)=\frac{z^{\nu}}{2^{\nu+1}} \int_{0}^{\infty} \frac{e^{-t-\frac{z^{2}}{4 t}}}{t^{\nu+1}} d t
$$

that corresponds to an integral representation for the modified Bessel function $K_{\nu}(z)$.
Taking $\nu=1$, replacing $z$ by $z Q$ and considering the following change of variable in the integral $t=Q^{2} \frac{1-x}{4 x}$, we obtain

$K_{1}(Q z)=\frac{z}{Q} \int_{0}^{1} \frac{d x}{(1-x)^{2}} \exp \left(-\frac{Q^{2}(1-x)}{4 x}-\frac{z^{2} x}{1-x}\right)$
[1] S. J. Brodsky and G. F. de Teramond, Phys. Rev. Lett. 96, 201601 (2006) arXiv:hep-ph/0602252.

[2] S. J. Brodsky and G. F. de Teramond, arXiv:0802.0514 [hep-ph]; G. F. de Teramond and S. J. Brodsky, AIP Conf. Proc. 1257, 59 (2010) arXiv:1001.5193 [hep-ph]].

[3] S. J. Brodsky and G. F. de Teramond, Phys. Lett. B 582, 211 (2004) arXiv:hep-th/0310227.

[4] S. J. Brodsky and G. F. de Teramond, Phys. Rev. D 77, 056007 (2008) arXiv:0707.3859 [hep-ph]].

[5] S. J. Brodsky and G. F. de Teramond, Phys. Rev. D 78, 025032 (2008) arXiv:0804.0452 [hep-ph]].

[6] A. Vega, I. Schmidt, T. Branz, T. Gutsche and V. E. Lyubovitskij, Phys. Rev. D 80, 055014 (2009) arXiv:0906.1220 [hep-ph]].

[7] A. Vega, I. Schmidt, T. Branz, T. Gutsche and V. E. Lyubovitskij, AIP Conf. Proc. 1265, 15 (2009) arXiv:1002.1518 [hep-ph]].

[8] T. Branz, T. Gutsche, V. E. Lyubovitskij, I. Schmidt and A. Vega, Phys. Rev. D 82, 074022 (2010) arXiv:1008.0268 [hep-ph]].

[9] A. Vega, I. Schmidt, T. Gutsche and V. E. Lyubovitskij, Phys. Rev. D 83, 036001 (2011) arXiv:1010.2815 [hep$\mathrm{ph}]$.

[10] A. Vega, I. Schmidt, T. Gutsche and V. E. Lyubovitskij, arXiv:1107.5553 [hep-ph].

[11] A. Vega, I. Schmidt, T. Gutsche and V. E. Lyubovitskij, arXiv:1109.6449 [hep-ph].

[12] T. Gutsche, V. E. Lyubovitskij, I. Schmidt and A. Vega, arXiv:1108.0346 [hep-ph].

[13] K. Goeke, M. V. Polyakov and M. Vanderhaeghen, Prog. Part. Nucl. Phys. 47, 401 (2001) arXiv:hep-ph/0106012.

[14] X. Ji, Ann. Rev. Nucl. Part. Sci. 54, 413 (2004).

[15] X. D. Ji, Phys. Rev. D 55, 7114 (1997) arXiv:hep$\mathrm{ph} / 9609381$.

[16] A. V. Radyushkin, Phys. Rev. D 56, 5524 (1997) arXiv:hep-ph/9704207.

[17] M. Diehl, T. Feldmann, R. Jakob and P. Kroll, Eur. Phys. J. C 39, 1 (2005) arXiv:hep-ph/0408173.

[18] M. Guidal, M. V. Polyakov, A. V. Radyushkin and M. Vanderhaeghen, Phys. Rev. D 72, 054013 (2005) arXiv:hep-ph/0410251.
[19] O. V. Selyugin and O. V. Teryaev, Phys. Rev. D 79, 033003 (2009) arXiv:0901.1786 [hep-ph]].

[20] J. Polchinski and M. J. Strassler, Phys. Rev. Lett. 88, 031601 (2002) arXiv:hep-th/0109174; H. Boschi-Filho and N. R. F. Braga, JHEP 0305, 009 (2003) arXiv:hepth/0212207; ; G. F. de Teramond and S. J. Brodsky, Phys. Rev. Lett. 94, 201601 (2005) arXiv:hepth/0501022 ; G. F. de Teramond and S. J. Brodsky, Phys. Rev. Lett. 102, 081601 (2009) arXiv:0809.4899 [hep-ph]]; J. Erlich, E. Katz, D. T. Son and M. A. Stephanov, Phys. Rev. Lett. 95, 261602 (2005) arXiv:hep-ph/0501128; L. Da Rold and A. Pomarol, Nucl. Phys. B 721, 79 (2005) arXiv:hep-ph/0501218; K. Ghoroku, N. Maru, M. Tachibana and M. Yahiro, Phys. Lett. B 633, 602 (2006) arXiv:hep-ph/0510334; E. Katz, A. Lewandowski and M. D. Schwartz, Phys. Rev. D 74, 086004 (2006) arXiv:hep-ph/0510388; H. R. Grigoryan and A. V. Radyushkin, Phys. Rev. D 76, 115007 (2007) arXiv:0709.0500 [hep-ph]].

[21] H. R. Grigoryan and A. V. Radyushkin, Phys. Lett. B 650, 421 (2007) arXiv:hep-ph/0703069.

[22] Z. Abidin and C. E. Carlson, Phys. Rev. D 79, 115003 (2009) arXiv:0903.4818 [hep-ph]].

[23] A. Vega and I. Schmidt, Phys. Rev. D 79, 055003 (2009) arXiv:0811.4638 [hep-ph]].

[24] A. Vega and I. Schmidt, AIP Conf. Proc. 1265, 226 (2010) arXiv:1002.1521 [hep-ph]].

[25] A. V. Radyushkin, Phys. Rev. D 58, 114008 (1998) arXiv:hep-ph/9803316.

[26] M. Abramowitz and I. Stegun, eds. Handbook of Mathematical Functions with Formulas, Graphs, and Mathematical Tables, New York: Dover Publications, 1972, $470 \mathrm{p}$.

[27] http://functions.wolfram.com/03.02.07.0004.01

[28] M. Burkardt, Phys. Rev. D 62, 071503 (2000) [Erratumibid. D 66, 119903 (2002)] arXiv:hep-ph/0005108.

[29] M. Burkardt, Int. J. Mod. Phys. A 18, 173 (2003) arXiv:hep-ph/0207047.

[30] G. A. Miller, Phys. Rev. Lett. 99, 112001 (2007) arXiv:0705.2409 [nucl-th]]. 